\title{
Non-Destructive Quality Analysis of JIRASAR Oryza Sativa SSP Indica (Indian Rice) using Feed Forward Neural Network
}

\author{
Niky K. Jain \\ Department of M Sc. (IT) \\ ISTAR, V.V.Nagar \\ Research Scholar RKU, Rajkot
}

\author{
Samrat O. Khanna \\ Dean School of Engineering \\ and Applied Science \\ Rai University
}

\author{
Chetna K. Shah \\ Department of Electronics and \\ Communication \\ GCET
}

\begin{abstract}
The Carrying out compelling and reasonable agriculture product has turned into an important issue in recent years. Agricultural production needs to stay aware with an everincreasing population. A key to this is the utilization of present day strategies (for precision agriculture) to exploit the quality in the market. Classification of rice seeds from the exposed human hands is neither savvy nor prescribed. The automatic grading for examination of quality has turned into the need of great importance. This paper prescribes an extra way to deal with quality specialists for the quality investigation of INDIAN JIRASAR Rice using computer vision and soft computing techniques. Computer Vision gives a grading methodology, non-destructive technique, along with multi-layer feed forward neural networking which achieves high degree of quality than human vision inspection.
\end{abstract}

\section{Keywords}

Computer Vision, Soft Computing technique, digital image processing, Indian Jirasar rice seeds, non-destructive.

\section{INTRODUCTION}

Indian agricultural production and development is thought to be excessively most seasoned and most broad in the entire world. In this time of Digital India where hi-tech upbringing of an agricultural industry has turned out to be more intelligent and automatic machinery has supplanted the human endeavours [1]. In India the opportunity has already come and gone to defeat the need of always expanding interest of generation by advancement in agricultural sector. Because of computerization need of high quality and safety standards achieved with accuracy, alongside quick and financially savvy quality assurance of agricultural products has expanded a great deal [11]. Quality being a noteworthy thought in any sustenance item is food product is determined from its physiochemical properties by human sensory panel which is tedious, may be varying results and costly as well as destructive chemical analysis [10]. Significant developments have been made in this field of computer vision since past few years [4]. Endeavors are being adapted towards the substitution of customary human tangible board with mechanized frameworks, as human operations are conflicting and less effective [5].

Oryza Sativa L. (Rice) is a fundamental overall agriculture product. It is one of the main sustenance yields of the world as the greater part of the total populace depends on rice as the real day by day wellspring of calories and protein. Rice (Oryza Sativa L) is developed in few nations such as India, China, Indonesia, Bangladesh and Thailand which are considered as the real makers. India is the world's second biggest producer and purchaser nation of rice for quite a while. It is one of the main nourishment harvests of the world as the greater part of the total populace depends on rice as the significant day by day wellspring of calories and protein [15].

The research work explained in this paper concentrates on the issue confronted by Indian Rice industry and its cost effective solution. Second section contains the specific issue of value assessment of Jirasar Rice seeds. The following segment examines about the different proposed philosophy being utilized alongside the materials for computing parameters for the nature of rice seeds. The proposed framework and additionally proposed calculation for processing Rice seeds with long, small and normal seeds classification is also discussed in the same section. Section 4 focus on the measurement for the quality of rice seeds based on computer vision handling and investigation. Section 5 indicates classification of rice seeds using multi-layer feed forward neural network for quality evaluation. Section 6 provides the conclusion of the proposed process.

\section{PROBLEM DEFINITION}

Foreign elements in terms of long as well as small seed as shown in Figure 1 have included in Jirasar rice (Oryza Sativa L) seeds. These seeds are having particularly significance in evaluating quality. At the time of handling these seeds are removed. Appropriate evacuation of this seed is fundamental in the event that it is not all that then it makes corruption in nature of rice seed. This paper proposes another strategy for tallying the quantity of Jirasar rice (Oryza Sativa L) seeds with these foreign elements as shown in Figure $2 a$ and $b$ using non-destructive technique based on artificial neural network to quantify the quality of Jirasar rice (Oryza Sativa L) seeds.

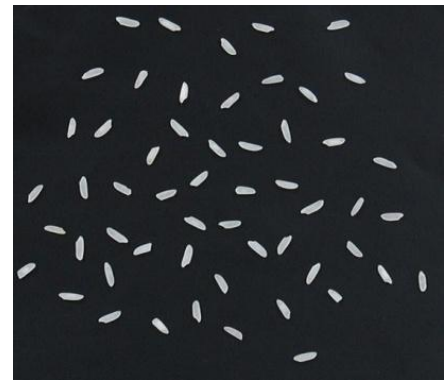

Fig. 1 Rice seeds with and without foreign elements

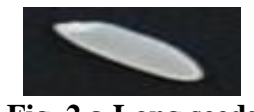

Fig. 2 a Long seeds present in the sample

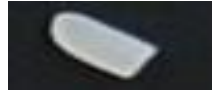

Fig. 2 b Foreign elements in the sample

\section{MATERIALS AND METHODS}

In this section we talk about the proposed algorithm. Here we have used different varietal samples of Jirasar rice. We characterize quality in view of the consolidated estimation method. We use minor axis length, major axis length, eccentricity, area, convex area, perimeter and extent of rice seed for counting the number of Jirasar rice (Oryza sativa L) seeds with long seeds, normal seeds as well as little seeds. 


\subsection{System Description and Operating Procedure}

A schematic outline of the proposed framework is in Figure 3. In our proposed framework there is a camera which is mounted on the highest point of the box at point 1 in Figure 3. The camera is having 12 mega pixels quality with $8 \mathrm{X}$ optical zoom. Subsequent to catching pictures of rice seed by camera is put away for additionally preparing.

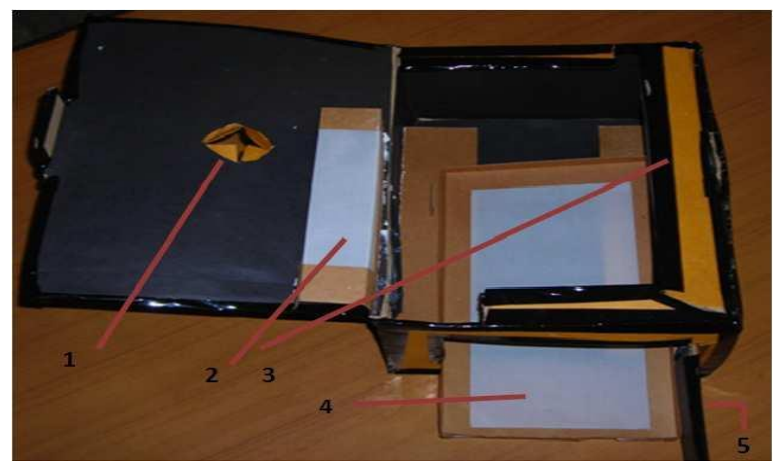

Fig. 3 Proposed Computer vision System for Analysis of Rice Seeds

The simplicity of operation of system can be concluded from the operating procedure detailed in Table 1 .

Table 1 Operating Procedure for Proposed System

\begin{tabular}{|c|l|}
\hline Sr. No. & STEPS \\
\hline $\mathbf{1}$ & $\begin{array}{l}\text { Consistently spread the specimen of seeds on } \\
\text { the plate without covering each other. }\end{array}$ \\
\hline $\mathbf{2}$ & Catch picture of seeds shape the framework \\
\hline $\mathbf{3}$ & Preparing and examination of caught picture \\
\hline $\mathbf{4}$ & $\begin{array}{l}\text { Show number of normal rice seeds, long seeds } \\
\text { and little seeds. }\end{array}$ \\
\hline $\mathbf{5}$ & Repeat above steps for 10 to 15 samples \\
\hline
\end{tabular}

\subsection{Proposed calculation to distinguish rice seeds with long and little seeds}

According to our calculation initially catch picture of test spread on the dark or butter paper utilizing camera.

Table 2 Proposed Algorithm

\begin{tabular}{|l|l|}
\hline Sr. No. & STEPS \\
\hline $\mathbf{1}$ & $\begin{array}{l}\text { Select the district of enthusiasm of the rice } \\
\text { seeds }\end{array}$ \\
\hline $\mathbf{2}$ & Convert over the RGB picture to dim picture. \\
\hline $\mathbf{3}$ & Apply the edge recognition operation. \\
\hline $\mathbf{4}$ & $\begin{array}{l}\text { Figure the parameters of the rice seeds. } \\
\text { Register the histogram of the parameters of } \\
\text { rice seeds and discover the threshold ranges. }\end{array}$ \\
\hline $\mathbf{5}$ & $\begin{array}{l}\text { Prepare the soft computing procedure for } \\
\text { classifying normal, long and little rice seeds. }\end{array}$ \\
\hline $\mathbf{7}$ & $\begin{array}{l}\text { Test Rice seeds of obscure specimen for } \\
\text { tallying ordinary, long and little rice seeds. }\end{array}$ \\
\hline
\end{tabular}

This image is colored in nature so we convert it into dim image as the color information is not of significance. The recognizable proof of articles inside of an image is an extremely troublesome errand. One approach to make direct the issue is to utilize ideal edge locator, ISEF [10], for extricating edges of dark scale picture. This stage recognizes singular item limits and denote the centre of every article for further handling. Thresholding is utilized to change over the portioned picture to a paired picture. The yield twofold picture has quality 1 (White territories) for all pixels of edges and 0 (dark) for every single other pixel.

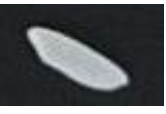

a

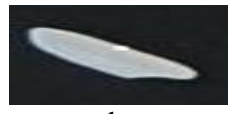

b

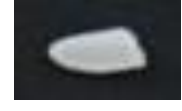

Fig. 4 Jirasar Rice seeds with and without foreign elements

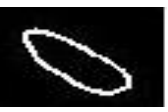

a

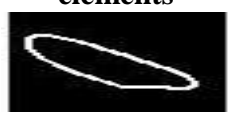

b

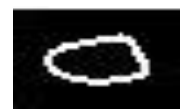

c
Fig. 5 After edge detection operation Jirasar Rice seeds without and with foreign elements

Normal rice seed appeared in figure 4(a),long seed appeared in figure 4(b) and small seed appeared in figure 4(c). Applying edge recognition operation on 4(a)-4(c) rice seed and after we get pictures of figures 5(a),(b) and(c) individually.

\subsection{Parameter Calculation}

Here we are extracting seven geometrical features namely minor axis length, major axis length, eccentricity, area, convex area, perimeter and extent for differentiating normal rice seed from long seed as well as little seed.

"The minor axis length $\mathrm{M}$ of a picture is characterized as the length (in pixels) of the minor hub of the circle that has the same standardized second focal minutes as the district."

"The major axis length $\mathrm{N}$ of a picture is characterized as the length (in pixels) of the real hub of the circle that has the same standardized second focal minutes as the locale."

"The eccentricity $\mathrm{E}$ is the ratio of the distance between the foci of the ellipse and its major axis length. The value is between 0 and 1 ."

"The area A of any object in an image is characterized by the aggregate number of pixels encased by the limit of the object."

"The Convex Area (co) of any object in an image is defined by scalar that specifies the number of pixels in image. This property is supported only for 2-D input label matrices."

"The Perimeter (pe) of any object in an image is defined by a path that surrounds a two dimensional shape. It can be thought of as the length of the diagram of a shape. The edge of a circle or oval is called its outline."

"The Extent (ex) of any object in an image is defined by scalar that specifies the ratio of pixels in the region to pixels in the total bounding box. It can be computed as the area divided by the area of the bounding object."

Encircled number 1, 2, 3 represents major axis of a small seed, normal seed and long seed respectively used for major axis calculation. Figure $6(\mathrm{a}-\mathrm{g})$ represents histograms for Area, Major Axis Length, Minor Axis Length, Eccentricity, Convex Area, Perimeter and Extent. 


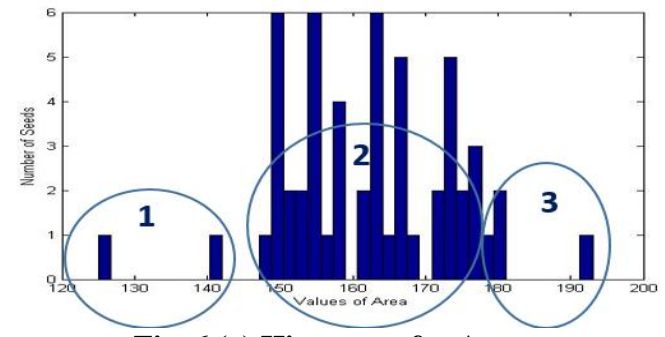

Fig. 6 (a) Histogram for Area

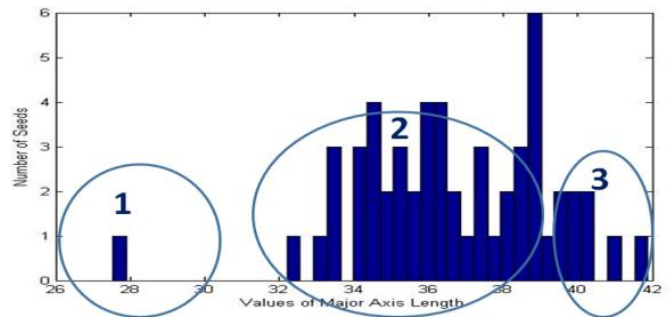

Fig. 6 (b) Histogram for Major Axis Length

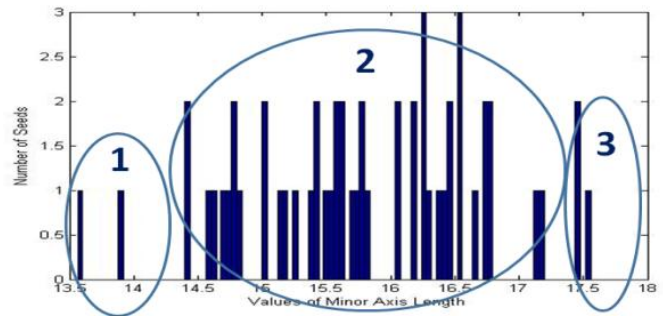

Fig. 6 (c) Histogram for Minor Axis Length

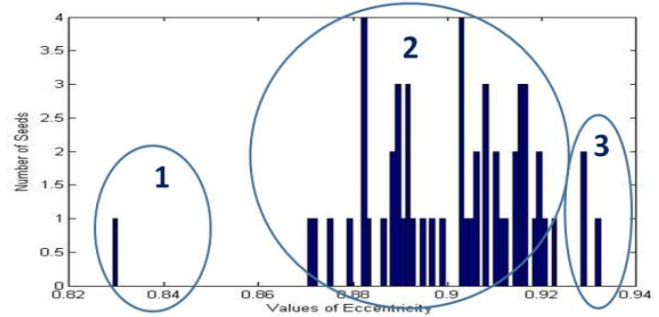

Fig. 6 (d) Histogram for Eccentricity

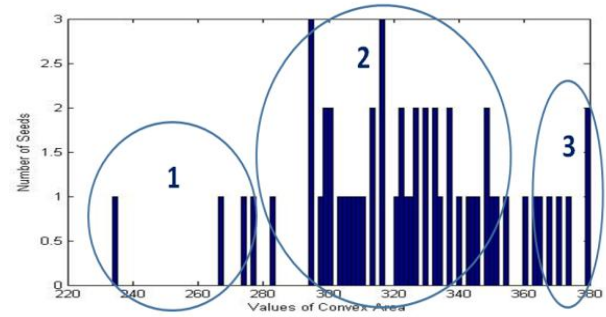

Fig. 6 (e) Histogram for Convex Area

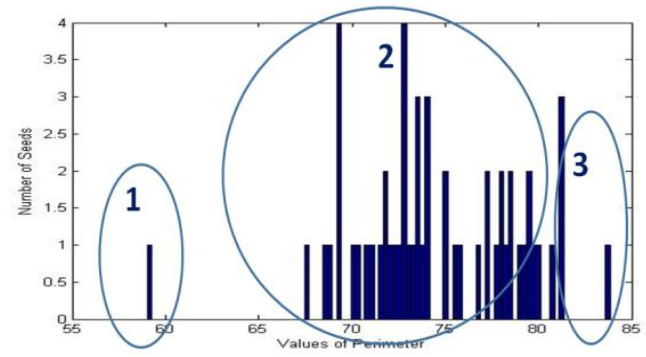

Fig. 6 (f) Histogram for Perimeter

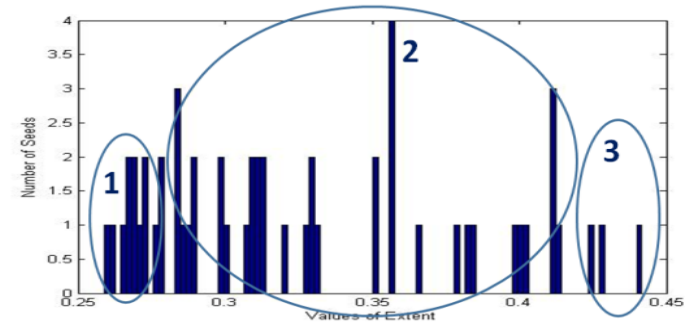

Fig. 6 (g) Histogram for Extent

\section{RESULTS ANALYSIS}

Table 3 represents parametric values based on histogram for all the three classification categories namely normal seeds, long seeds and small seeds respectively. The values in the table displays the, Area (A), Major axis length (MJ), Minor axis length (MN), Eccentricity (E), Convex area (CO), Perimeter (PE) and Extent (EX) of various rice seeds available in one sample. Same way values of all seven parameters of 25 samples are found where each sample contains approximately 50 seeds.

Table 3 Analysis for 15 random seeds Available in One

\begin{tabular}{|c|c|c|c|c|c|c|c|}
\hline \multicolumn{1}{c|}{ Sample } \\
\hline 1 & 155 & 36.45 & 17.17 & 0.88 & 333 & 74.08 & 0.25 \\
\hline 2 & 152 & 36.47 & 14.76 & 0.91 & 299 & 71.84 & 0.27 \\
\hline 3 & 149 & 35.09 & 13.89 & 0.91 & 277 & 70.28 & 0.38 \\
\hline 4 & 180 & 40.03 & 14.83 & 0.92 & 340 & 80.04 & 0.42 \\
\hline 5 & 150 & 32.41 & 14.62 & 0.89 & 274 & 67.45 & 0.41 \\
\hline 6 & 177 & 38.83 & 17.55 & 0.89 & 370 & 78.04 & 0.41 \\
\hline 7 & 178 & 35.76 & 16.31 & 0.88 & 333 & 74.04 & 0.44 \\
\hline 8 & 165 & 38.31 & 15.68 & 0.91 & 337 & 77.35 & 0.31 \\
\hline 9 & 173 & 38.98 & 16.75 & 0.90 & 361 & 78.91 & 0.26 \\
\hline 10 & 167 & 39.10 & 15.76 & 0.91 & 343 & 78.18 & 0.29 \\
\hline 11 & 193 & 40.17 & 15.54 & 0.92 & 365 & 81.11 & 0.37 \\
\hline 12 & 177 & 40.20 & 17.11 & 0.90 & 379 & 81.25 & 0.26 \\
\hline 13 & 155 & 34.46 & 15.26 & 0.89 & 297 & 70.18 & 0.31 \\
\hline 14 & 174 & 39.73 & 16.78 & 0.90 & 374 & 81.11 & 0.32 \\
\hline 15 & 155 & 34.09 & 16.52 & 0.87 & 313 & 72.28 & 0.33 \\
\hline
\end{tabular}

For classification of the three categories we compute thresholds values using the histograms of Figure $6(\mathrm{a}-\mathrm{g})$. Table 4 mentions all the seven parameters and there computed threshold using histogram for area, major axis length, minor axis length, eccentricity, convex area, perimeter and extent.

Table 4 Computed Threshold Values

\begin{tabular}{|c|c|c|c|}
\hline Parameters & $\begin{array}{c}\text { Small } \\
\text { seed }\end{array}$ & $\begin{array}{c}\text { Normal } \\
\text { seed }\end{array}$ & Long seed \\
\hline A & $110-140$ & $140-180$ & $180-200$ \\
\hline MJ & $25-31$ & $31-39$ & $39-45$ \\
\hline MN & $10-14$ & $14-17$ & $17-25$ \\
\hline E & $0.65-0.84$ & $0.84-0.93$ & $0.93-1.00$ \\
\hline CO & $200-270$ & $270-390$ & $390-410$ \\
\hline PE & $50-66$ & $66-82$ & $82-90$ \\
\hline EX & $0.2-0.25$ & $0.25-0.45$ & $0.45-0.50$ \\
\hline
\end{tabular}




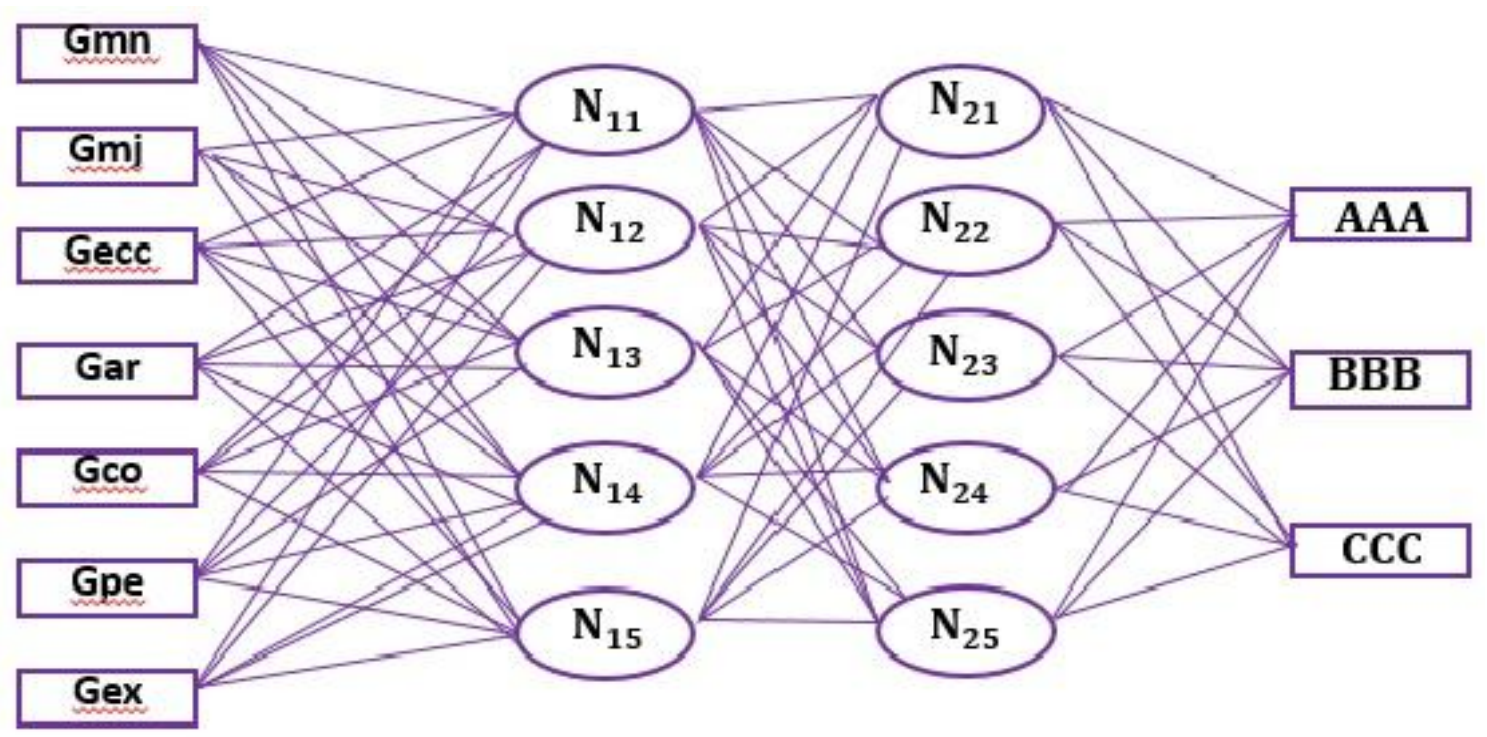

Fig. 7 Trained two layer feed forward neural network of Jirasar rice seeds of 15 samples Schematic diagram

Table 5 Grading Of Several Seeds Based On Priority of Parameters Available In One Sample

\begin{tabular}{|c|c|c|c|c|c|c|c|c|c|c|c|c|c|c|c|}
\hline $\begin{array}{c}\text { S. } \\
\text { No }\end{array}$ & $\mathbf{m n}$ & $\underset{\mathbf{n}}{\mathbf{G m}}$ & $\mathbf{m j}$ & Gmj & ecc & Gecc & ar & Gar & Co & Gco & PE & Gpe & Ex & Gex & Grade \\
\hline 1 & 17.17 & B & 36.45 & B & 0.88 & B & 155 & B & 333 & B & 74.08 & B & 0.25 & B & BBB \\
\hline 2 & 14.76 & B & 36.47 & B & 0.91 & B & 152 & B & 299 & B & 71.84 & B & 0.27 & B & BBB \\
\hline 3 & 13.89 & A & 35.09 & B & 0.64 & A & 139 & A & 277 & B & 65.28 & A & 0.38 & B & AAA \\
\hline 4 & 14.83 & B & 40.03 & C & 0.92 & B & 180 & B & 340 & B & 80.04 & B & 0.42 & B & BBB \\
\hline 5 & 14.62 & B & 32.41 & B & 0.89 & B & 150 & B & 274 & B & 65.45 & A & 0.41 & B & BBB \\
\hline 6 & 17.55 & $\mathrm{C}$ & 38.83 & B & 0.89 & B & 177 & B & 370 & B & 78.04 & B & 0.41 & B & BBB \\
\hline 7 & 16.31 & B & 35.76 & B & 0.88 & B & 178 & B & 333 & B & 74.04 & B & 0.44 & B & BBB \\
\hline 8 & 13.68 & A & 28.31 & A & 0.91 & B & 135 & A & 237 & A & 77.35 & B & 0.31 & B & AAA \\
\hline 9 & 16.75 & B & 38.98 & B & 0.90 & B & 173 & B & 361 & B & 78.91 & B & 0.26 & B & BBB \\
\hline 10 & 15.76 & B & 39.10 & C & 0.91 & B & 167 & B & 343 & B & 78.18 & B & 0.29 & B & BBB \\
\hline
\end{tabular}

\section{CLASSIFICATION}

\subsection{NEURAL NETWORK (NN)}

A two layer feed forward neural network with 5 nodes in each layer is used for classification. The training function using gradient descent with momentum weight as learning function, and maximum likelihood as a performance function is used to train the NN. Gar,Gmj, Gmn, Gecc,Gco,Gpe and Gex symbolises grades of Area,Major axis length, Minor axis length, Eccentricity,Convex area,Perimeter and Extent respectively as shown in figure $7 . \mathrm{N} 11, \mathrm{~N} 12, \mathrm{~N} 13, \mathrm{~N} 14$ and N15 are nodes in Hidden layer 1 while N21, N22, N23, N24 and N25 are nodes in Hidden layer 2. Output labels are AAA, $\mathrm{BBB}$ and CCC. AAA is assigned for small seed, BBB for normal and $\mathrm{CCC}$ for large seed.

The training of the Neural Network was performed using 15 samples containing 750 Jirasar rice seeds and later an unknown sample consisting of random rice seeds of different quality in bulk was tested. For training the Neural Network, rice seeds in images are divided into three classes i.e. large quality, Normal quality and Small quality by taking into consideration all the seven parameters namely minor axis, major axis, eccentricity, area, convex area, perimeter and extent limit shown in table 4.

Table 5 displays Gmn, Gmj, Gecc, Gar, Gco, Gpe and Gex are grades of Minor Axis Length, Major Axis Length, Eccentricity, Area, Convex Area, Perimeter and Extent parameters respectively. The table provides grading of several Jirasar rice seeds of one sample. Here we had defined parameter of a small seed as $\mathbf{A}$, normal seed as $\mathbf{B}$ and large seed as $\mathbf{C}$. The priorities of parameters are minor axis, major axis, eccentricity, area, convex area, perimeter and extent. Grades of these seven parameters based on priority would finally decide the Grade of Jirasar rice seeds. 
Training dataset is prepared for Multilayer feed forward neural network on the basis of Table $4 \& 5$ respectively. And then we have trained neural network for dataset of 15 samples of Jirasar rice seeds as shown in figure 7. Table 6 shows all parameters used for training of multi-layer feed forward neural network which we have used for classification.

Table 6 Parameters Used For Neural Network In XL Miner

\begin{tabular}{|c|c|}
\hline \multicolumn{2}{|c|}{ Parameters / Options } \\
\hline Cost Function & Maximum Likelihood \\
\hline Hidden Layer Sigmoid & Standard \\
\hline Output Layer Sigmoid & Standard \\
\hline Epochs & 30 \\
\hline Step size for Gradient descent & 0.1 \\
\hline Weight change momentum & 0.6 \\
\hline Error Tolerance & 0.01 \\
\hline
\end{tabular}

When the training was finished using neural networking the network was tested with unknown sample. The classification accuracy of test dataset is $97 \%$.

\section{CONCLUSION}

Quality quantification of Krishna Kamod rice seeds using computer vision analysis and soft computing techniques have given an advancement in the agricultural sector of India. Here we were able to calculate geometrical features like minor axis length, major axis length, eccentricity and area for counting normal seed and foreign element in terms of long as well as small seed for a given sample. A new technology with nondestructive quality analysis of rice seeds using feed forward neural network provides an accuracy of $97 \%$ and was quiet effectively used in the rice mills of nearby areas. Traditionally quality evaluation and assessment is done by human sensory panel which is time consuming and costly.

\section{REFERENCES}

[1] Abdullah MZ, Fathinul-Syahir AS, Mohd-Azemi BMN, "Automated inspection system for color and shape grading of star fruit (Averrhoa carambola L.) using Computer vision sensor," Transactions of the Institute of Measurement and Control, 27 (2), 65-87, 2005.

[2] Abutaleb AS, "Automatic thresholding of grey-level pictures using two-dimensional entropies." Pattern Recognition, 47(1), 22-32, 1989.
[3] Ballard, D. A., \& Brown, C. M. " Computer vision", Englewood Cliffs, NJ, USA: Prentice-Hall ,1982.

[4] Blasco J, Aleixos N, Molt E, "Computer vision system for automatic quality grading of fruit", Biosystems Engineering, 415-423, 2003.

[5] Chetna Maheshwari, Kavindra Jain, Chintan Modi, "Novel approach for Oryza sativa L.(Rice) based on Computer vision technology," PEPCCI, National Conference ,ISBN No.-978-93-81286-06-7,2012.

[6] Du CJ, Sun D-W, "Recent development in the applications of image processing techniques for food quality evaluation." Trends in Food Science and Technology, 15,230-249, 2004.

[7] Du C-J, Sun D-W, "Learning techniques used in computer vision for food quality evaluation: a review", Journal of Food Engineering, 72(1), 39-55, 2006.

[8] Gunasekaran Sundaram, Kexiang Ding, "Computer vision technology for food quality assurance,". Trends in Food Science and Technology, 7, 245-256, 1996.

[9] Jain AK, "Fundamentals of Digital Image Processing," Englewood Cliffs: Prentice-Hall 1989.

[10] Kavindra Jain, Chintan K. Modi, Kunal Pithadiya, "Non Destructive quality evaluation in spice industry with specific reference to Cuminum Cyminum L (Cumin) seeds," International Conference on Innovations \& Industrial Applications, Malaysia, (IEEE) 2009.

[11] M. Kurita and N. Kondo, "Agricultural product grading method by image processing (part 1) - effectiveness of direct lighting method", J.SHITA 18(1): 9-17,2006.

[12] Shen Castan, Sian Zhao,"A Comparitive study of Performance of Noisy roof edge detection", 5th International conference on Computer analysis of Images and Patterns, volu.179, pp 170-174

[13] Tadhg Brosnan, Da-Wen Sun, "Improving quality inspection of food products by computer vision-a review", Journal of Food Engineering 61, pp. 3-16, 2004.

[14] Xiaopei Hu, ParmeshwaraK.M, DavidV. "Development of Non Destructive Methods To Evaluate Oyster Quality By Electronic Nose Technology", Springer Science Business Media, LLC, 2008.

[15] Niky K. Jain,Samrat O. Khanna,Chetna Maheshwari,,"Feed Forward Neural Network Classification for INDIAN Krishna Kamod Rice'International Journal of Computer Applications(0975-8887),Volume 134 - No. 14,January 2016 